\title{
SOME FIBRED COBORDISM GROUPS ARE NOT FINITELY GENERATED
}

\author{
L. ASTEY, P. GREENBERG, E. MICHA AND G. PASTOR \\ (Communicated by Haynes R. Miller)
}

\begin{abstract}
By showing that certain Pontrjagin classes are fibre cobordism invariants "of connected type" we produce infinitely many examples of fibre cobordism groups which are not finitely generated.
\end{abstract}

1. Introduction. If $X$ is a closed manifold we shall denote by $N_{p}^{X}$ the cobordism group of smooth fibrations over $X$ with fibre $p$-dimensional closed manifolds. Here a cobordism between two such fibrations $q_{1}: Z_{1} \rightarrow X$ and $q_{2}: Z_{2} \rightarrow X$, with fibres $M_{1}$ and $M_{2}$ respectively, is a smooth fibration $q: W \rightarrow X$ with fibre a compact manifold $N$, such that $\partial W=Z_{1} \amalg Z_{2}, \partial N=M_{1} \amalg M_{2}$ and the restriction of $q$ to $\partial W$ is $q_{1} \amalg q_{2}$. In the usual manner cobordism classes form an abelian group under the disjoint union.

The oriented version of these groups has been studied by M. Kreck [K], P. Melvin $[\mathbf{M}]$ and F. Bonahon [B] when $X$ is a circle. A. Didierjean [D1] computed the groups $N_{0}^{X}, N_{1}^{X}$ and showed that, if $X$ is simply connected, any nonzero element of $N_{2}^{X}$ can be represented by a fibration with fibre a projective plane $\mathbf{R} P^{2}$.

In this note we shall only consider $X$ to be a sphere of dimension $4 n$, and construct for some cobordism classes invariants of "connected type" arising from certain Pontrjagin classes. These invariants will allow us to deduce that some of these cobordism groups are not finitely generated and, in particular, to compute $N_{2}^{S^{4}}$. We wish to express our thanks to A. Didierjean for bringing the question of invariants of connected type to our attention [D2].

2. Pontrjagin classes as an invariant of connected type. Let $n$ be a positive integer. In this section we will only consider $(4 n-1)$-dimensional real vector bundles over the sphere $S^{4 n}$. If $\gamma$ is a vector bundle we will denote by $S(\gamma)$, $D(\gamma)$ and $P(\gamma)$ the associated sphere, disc and projective bundles. The tangent bundle of $S(\gamma)$ is then stably equivalent to $\pi^{*}(\gamma)$, where $\pi: S(\gamma) \rightarrow S^{4 n}$ denotes the projection. Looking at the Gysin sequence one finds that $\pi^{*}: H^{4 n}\left(S^{4 n}, \mathbf{Z}\right) \rightarrow$ $H^{4 n}(S(\gamma) ; \mathbf{Z})$ is an isomorphism and therefore we obtain

LEMMA. If $\gamma$ is a $(4 n-1)$-vector bundle over $S^{4 n}$, then the isomorphism $\pi^{*}$ : $H^{4 n}\left(S^{4 n} ; \mathbf{Z}\right) \rightarrow H^{4 n}(S(\gamma) ; \mathbf{Z})$ sends the nth-Pontrjagin class $p_{n}(\gamma)$ into $p_{n}(S(\gamma))$.

Let $\gamma_{1}$ and $\gamma_{2}$ be $(4 n-1)$-vector bundles over $S^{4 n}$. Assume further that there is a connected cobordism $W$, fibring over $S^{4 n}$ with $\partial W=S\left(\gamma_{1}\right) \amalg S\left(\gamma_{2}\right)$. The fibre of the cobordism is then a $(4 n-1)$-compact manifold $N$ with boundary two copies of the sphere $S^{4 n-2}$. Since $N$ has the homotopy type of a $(4 n-2)$-complex,

Received by the editors September 30, 1987.

1980 Mathematics Subject Classification (1985 Revision). Primary 57R90; Secondary 57R20. 
looking at the spectral sequence of the fibration, one finds that the projection $W \rightarrow S^{4 n}$ induces also an isomorphism $H^{4 n}\left(S^{4 n} ; \mathbf{Z}\right) \rightarrow H^{4 n}(W ; \mathbf{Z})$. We have then a commutative diagram

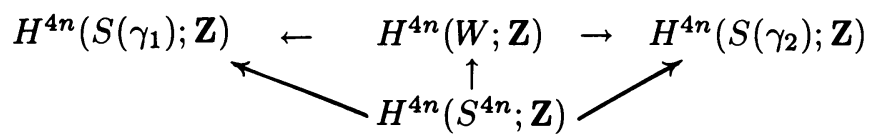

where all arrows, induced either by inclusions or projections, are isomorphisms. Since the Pontrjagin class $p_{n}(W)$ restricts to $p_{n}\left(S\left(\gamma_{i}\right)\right)$, the lemma now implies

THEOREM 1. Let $S\left(\gamma_{1}\right)$ and $S\left(\gamma_{2}\right)$ be sphere bundles associated to the $(4 n-1)$ vector bundles $\gamma_{1}$ and $\gamma_{2}$ over the sphere $S^{4 n}$. Assume further that there is a connected cobordism $W$ fibring over $S^{4 n}$ with $\partial W=S\left(\gamma_{1}\right) \amalg S\left(\gamma_{2}\right)$. Then $\gamma_{1}$ and $\gamma_{2}$ are isomorphic as vector bundles.

Observe that any $S(\gamma)$ represents the zero element in $N_{4 n-2}^{S^{4 n}}$, being the boundary of the disc bundle $D(\gamma)$. Our result asserts that if $\gamma_{1}$ and $\gamma_{2}$ are not isomorphic, then $S\left(\gamma_{1}\right)$ and $S\left(\gamma_{2}\right)$ are not fibred cobordant through a connected cobordism.

THEOREM 2. Let $\gamma_{1}$ and $\gamma_{2}$ be $(4 n-1)$-vector bundles over $S^{4 n}$. Then the projective bundles $P\left(\gamma_{1}\right)$ and $P\left(\gamma_{2}\right)$ are fibred cobordant if and only if $\gamma_{1}$ and $\gamma_{2}$ are isomorphic.

PROOF. First observe that since the fibre $\mathbf{R} P^{4 n-2}$ is not a boundary, $P(\gamma)$ represents a nonzero element in $N_{4 n-2}^{S^{4 n}}$, for any bundle $\gamma$. Therefore, if $P\left(\gamma_{1}\right)$ and $P\left(\gamma_{2}\right)$ are fibred cobordant, there is always a connected cobordism $W$, fibring over $S^{4 n}$ with $\partial W=P\left(\gamma_{1}\right) \amalg P\left(\gamma_{2}\right)$. But then, the double covering of $W$ associated to the orientation bundle provides a fibred cobordism between the sphere bundles $S\left(\gamma_{1}\right)$ and $S\left(\gamma_{2}\right)$. Theorem 1 now implies that $\gamma_{1}$ and $\gamma_{2}$ are isomorphic.

COROLLARY. for $n \geq 1$, the groups $N_{4 n-2}^{S^{4 n}}$ are not finitely generated.

PROOF. The isomorphism classes of $(4 n-1)$-vector bundles over $S^{4 n}$ are classified by the set of homotopy classes $\left[S^{4 n}, \mathrm{BSO}(4 n-1)\right]$, which is infinite.

Finally we consider the group $N_{2}^{S^{4}}$. According to [D1] one knows that there is an epimorphism $\phi: \bigoplus \mathbf{Z}_{2} \rightarrow N_{2}^{S^{4}}$ where the direct sum is indexed by $\left[S^{4}, \mathrm{BSO}(3)\right]$. We conclude from Theorem 2 that $\phi$ is in fact an isomorphism.

\section{REFERENCES}

[B] F. Bonahon, Cobordisme des difféomorphismes de surfaces, C. R. Acad. Sci. Paris Sér. A-B 290 (1980), A765-A767.

[D1] A. Didierjean, Cobordisme fibré et approximation d'une sous-variété singulière par des sousvariété $C^{\infty}$, Ann. Inst. Fourier (Grenoble) 33 (1983), 277-306.

[D2] - Cobordisme fibré connexe, preprint.

[K] M. Kreck, Bordism of diffeomorphisms and related topics, Lecture Notes in Math., vol. 1069, Springer-Verlag, Berlin and New York, 1984.

[M] P. Melvin, Bordism of diffeomorphisms, Topology 18 (1979), 173-175.

Departamento de Matemáticas, Centro de Investigacion y de Estudios Avanzados Del I. P. N., Apartado Postal 14-740, MÉxico 07000, D. F. 